Pacific Journal of Mathematics

ANALYTIC PHENOMENA IN GENERAL FUNCTION

C. E. RICKART 


\title{
ANALYTIC PHENOMENA IN GENERAL FUNCTION ALGEBRAS
}

\author{
C. E. RICKART
}

Let $\Sigma$ be a locally compact Hausdorff space and $\mathfrak{A}$ an algebra of complex-valued continuous functions on $\Sigma$ which contains the constant functions. Assume that $\Sigma$ carries the weakest topology in which every function in $\mathfrak{A}$ is continuous and that each homomorphism of $\mathfrak{A}$ onto the complex numbers is given by evaluation at a point of $\Sigma$. Then $\mathscr{Y}$ is called a natural algebra of functions on $\Sigma$. The motivating example for most of this paper is the algebra $\mathscr{P}$ of all polynomials in $n$ complex variables. It is readily verified that $\mathscr{P}^{\circ}$ is in fact a natural algebra of functions on the $n$-dimensional complex space $C^{n}$. In the general setting an abstract analytic function theory is constructed for $\Sigma$ with the natural algebra $\mathfrak{A}$ playing a role analogous to that of $\mathscr{P}^{\circ}$ in the case of $C^{n}$. For example, the concepts of $\mathfrak{U}$-holomorphic functions and, in terms of these functions, $\mathscr{U}$-analytic varieties in $\Sigma$ are introduced. The first main result obtained is that every $\mathfrak{A}$ analytic subvariety of a compact $\mathfrak{A}$-convex subset of $\Sigma$ is itself $\mathfrak{X}$-convex. Next let $\Omega$ be any compact $\mathfrak{X}$-convex subset $\Sigma$ and $U$ a relatively open subset of $\Omega$ disjoint from the Šilov boundary of $\Omega$ with respect to $\mathscr{A}$. Consider a connected subset $\mathscr{F}$ of the space $C(\bar{U})$ of all complex-valued continuous functions on the closure $\bar{U}$ of the set $U$. Let each function in $\mathscr{F}$ be $\mathfrak{A}$-holomorphic in $U$ and assume that some but not all of the functions in $\mathscr{F}$ have zeros in $U$. Then the second main result is that $\mathscr{F}$ must contain a function with zeros on the topological boundary of $U$ relative to the space $\Omega$. This implies a local property of $\mathfrak{X}$-convex hulls which generalizes an important result due to $K$. Oka for polynomially convex hulls in $C^{n}$.

The theorems obtained below contain well-known results for $\boldsymbol{C}^{n}$ which depend more-or-less directly on the fundamental contributions made by K. Oka [5] to the theory of analytic functions of several complex variables. (See, for example, [9, (A. 21) p. 285] for convexity properties of subvarieties and [5, pp. 13, 14] or [9, (1.4) p. 264] for the local properties of convex hulls in $\boldsymbol{C}^{n}$.) The proofs of the general theorems are entirely in the spirit of function algebras and consequently are quite different from the usual proofs for $\boldsymbol{C}^{n}$. In fact, the theory of analytic functions of several complex variables is involved only indirectly through the use of $\mathrm{H}$. Rossi's local maximum modulus principle for general function algebras [8] whose proof does depend on several complex variable theory. 
In Section 1 a few notations, definitions and elementary properties of natural algebras of functions are introduced; Section 2 contains a discussion of functions that are holomorphic in an appropriate sense relative to a natural algebra; Section 3 contains the results on convexity of subvarieties; and Section 4 contains the local property of convex hulls mentioned above along with a few other related results.

1. Preliminaries. Let $\Sigma$ be a locally compact Hausdorff space. We will be concerned with complex-valued functions defined and continuous (but not necessarily bounded) on the space $\Sigma$ or on a subset thereof. The algebra operations involving such functions will always be the pointwise operations for functions. If $f$ is defined on a set $X \subseteq \Sigma$ and if $Y \subseteq X$, then the function obtained by restricting $f$ to the set $Y$ is denoted by $f \mid Y$. We also define

$$
|f|_{Y}=\sup _{\sigma \in Y}|f(\sigma)| \text {. }
$$

Let $\mathfrak{A}$ be an algebra of complex-valued continuous functions defined on all of $\Sigma$. We call such an algebra a natural algebra of functions on $\Sigma$ provided it contains the constant functions and has $\Sigma$ as its carrier space [6, p. 110]. The first condition means that $\mathfrak{A}$ has an identity element. The second condition amounts to the assumption that every homomorphism $\varphi$ of $\mathfrak{A}$ onto the complex numbers be of the form $\varphi: f \rightarrow f\left(\sigma_{\varphi}\right)$, where $\sigma_{\varphi}$ is a point of $\Sigma$, and that the topology in $\Sigma$ be the weakest under which all functions in $\mathfrak{A}$ are continuous $[6$, p. 110]. We call this topology the $\mathfrak{X}$-topology for $\Sigma$. It can also be defined as the topology determined by neighborhoods of the form

$$
V_{\sigma_{0}}=\left\{\sigma: \sigma \in \Sigma,\left|f_{i}(\sigma)-f_{i}\left(\sigma_{0}\right)\right|<1(i=1, \cdots, k)\right\},
$$

where $f_{1}, \cdots, f_{k}$ is an arbitrary finite set in $\mathfrak{A}$. It is evident from this remark that, if $\Sigma$ carries the $\mathfrak{A}$-topology and $X$ is an arbitrary subset of $\Sigma$, then the relative topology in $X$ coincides with the $\mathfrak{A} \mid X$ topology, where $\mathfrak{A} \mid X$ is the algebra of functions on $X$ obtained by restricting functions from $\mathfrak{A}$ to $X$. Note that elements of $\mathfrak{U}$ separate the points of $\Sigma$. If $\Sigma$ is compact, then the condition that $\mathfrak{A}$ separate the points of $\Sigma$ is sufficient as well as necessary for the topology in $\Sigma$ to be the $\mathfrak{A}$-topology.

Throughout the remainder of this paper, we will always require that $\mathfrak{A}$ be a natural algebra of functions on $\Sigma$, although in some places the full assumption is not needed.

As was remarked in the Introduction, the algebra $\mathscr{P}$ of all polynomials in $n$ complex variables is a natural algebra of functions on $\boldsymbol{C}^{n}[6$, p. 149]. Although this is the example that guides most of what we do, another important example of a natural function algebra 
is the algebra of functions obtained from the Gelfand representation of an arbitrary commutative Banach algebra with identity [6, p. 119]. In this case the space $\Sigma$ is compact, being the carrier space (space of maximal ideals) of the Banach algebra.

Let $X$ be an arbitrary subset of $\Sigma$. Then the set

$$
\left\{\sigma: \sigma \in \Sigma,|f(\sigma)| \leqq|f|_{x}, f \in \mathfrak{U}\right\}
$$

is called the $\mathfrak{A}$-convex hull of $X$ in $\Sigma$ and is denoted by hull $X$. If

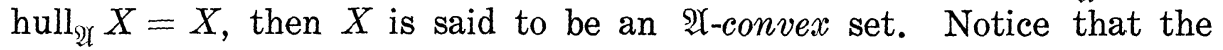
$\mathfrak{A}$-convex hull of a set $X$ is always closed, contains $X$, and is $\mathfrak{A}$ convex. Now let $\mathfrak{D}$ denote another algebra of continuous functions on $\Sigma$. Indicate by $\overline{\mathfrak{A}}$ the closure of $\mathfrak{A}$ in the topology of uniform convergence on compact subsets of $\Sigma$. If $X$ is compact and $\mathfrak{D} \subseteq \overline{\mathfrak{A}}$, then it is readily proved that

$$
\operatorname{hull}_{\mathfrak{A}} X \subseteq \operatorname{hull}_{\mathfrak{D}} X .
$$

Thus, if $\mathfrak{A}$ and $\mathfrak{D}$ have the same closure, then

$$
\operatorname{hull}_{\mathfrak{A}} X=\operatorname{hull}_{\mathfrak{D}} X \text {. }
$$

In particular, we have

$$
\operatorname{hull}_{\mathfrak{U}} X=\operatorname{hull}_{\overline{\mathfrak{T}}} X \text {. }
$$

Let $X$ be a compact set in $\Sigma$ and let $\Omega=\operatorname{hull}_{\mathfrak{Q}} X$. Then, for each $f \in \mathfrak{A}$, we have

$$
|f|_{\Omega}=|f|_{x}<\infty \text {. }
$$

Therefore $\mathfrak{A} \mid \Omega$ is a normed algebra. The completion of $\mathfrak{A} \mid \Omega$ under its norm can obviously be identified with the algebra $\mathfrak{A}_{\Omega}$ of continuous functions on $\Omega$ which are uniform limits on $\Omega$ of elements of $\mathfrak{A} \mid \Omega$. Evidently $\mathfrak{H}_{\Omega}$ is isometrically isomorphic with the algebra $\mathfrak{A}_{x}$ obtained by closing $\mathfrak{A} \mid X$ in the Banach algebra $C(X)$. The following lemma, which depends in an essential way on the assumption that $\mathfrak{A}$ be natural, will be used frequently below.

Lemma 1.1. Let $X$ be a compact set with $\mathfrak{A}$-convex hull $\Omega$. Then $\Omega$ is also compact and $\mathfrak{A}_{\Omega}$ is a natural algebra of functions on $\Omega$.

Proof. Denote the carrier space of $\mathfrak{A}_{\Omega}$ by $\Phi$. For each $\omega \in \Omega$ set

$$
\hat{f}\left(\varphi_{\omega}\right)=f(\omega) \quad \text { for } f \in \mathfrak{A}_{\Omega} .
$$

Then $\varphi_{\omega} \in \Phi$ and the mapping $\omega \rightarrow \phi_{\omega}$ is the natural embedding of $\Omega$ 
in $\Phi$. Since the topology in $\Omega$ is the $\mathfrak{A} \mid \Omega$-topology, it follows that the embedding is a homeomorphism. We show that $\Omega$ maps onto $\Phi$. Let

$$
\varphi: f \rightarrow \hat{f}(\varphi), \quad f \in \mathfrak{A}_{\Omega},
$$

be any homomorphism of $\mathfrak{A}_{\Omega}$ onto the complex numbers. Then the: mapping

$$
a \rightarrow a \mid \Omega \rightarrow(\widehat{a \mid \Omega})(\varphi) \text { for } a \in \mathfrak{U}
$$

defines a homomorphism of $\mathfrak{A}$ onto the complex numbers. Since $\mathfrak{A}$ is natural on $\Sigma$, there exists $\sigma_{\varphi} \in \Sigma$ such that

$$
(\widehat{a \mid \Omega})(\varphi)=a\left(\sigma_{\varphi}\right) \quad \text { for } a \in \mathfrak{A}
$$

Now $\mathfrak{A}_{\Omega}$ is a Banach algebra with norm $|f|_{\Omega}$ so we have

$$
|\hat{f}(\varphi)| \leqq|f|_{\Omega} \quad \text { for } f \in \mathfrak{A}_{\Omega} \text {. }
$$

Therefore, in particular,

$$
\left|a\left(\sigma_{\varphi}\right)\right| \leqq|a|_{\Omega}=|a|_{X} \quad \text { for } a \in \mathfrak{A} .
$$

This means that $\sigma_{\varphi} \in \Omega$. Since $\mathfrak{A} \mid \Omega$ is dense in $\mathfrak{A}_{\Omega}$, it follows that $\hat{f}(\varphi)=f\left(\sigma_{\varphi}\right)$ for all $f \in \mathfrak{A}_{\Omega}$. In other words, the image of $\Omega$ in $\Phi$ under the mapping $\omega \rightarrow \varphi_{\omega}$ exhausts $\Phi$. Therefore $\Omega$ and $\Phi$ are homeomorphic and $\mathfrak{A}_{\Omega}$ is a natural algebra of functions on $\Omega$. That $\Omega$ is compact follows from the fact that the carrier space of a Banach algebra with identity element is compact.

CoROLlaRY 1.2. Let $\Omega$ be an arbitrary compact $\mathfrak{A}$-convex subset of $\Sigma$. Then $\mathfrak{A}_{\Omega}$ is a natural algebra of function on $\Omega$.

Lex $X$ be a compact subset of $\Sigma$. A closed set $B \cong X$ is called a boundary of $X$ relative to $\mathfrak{A}$, or simply an $\mathfrak{A}$-boundary of $X$, if for every $f \in \mathfrak{A}$, it is true that

$$
\sup _{\sigma \in B}|f(\sigma)|=\sup _{\sigma \in X}|f(\sigma)| \text {. }
$$

There always exists a unique minimal $\mathfrak{A}$-boundary for $X$ which is called is Silov boundary of $X$ relative to $\mathfrak{A}$ and denoted by $\partial_{\mathfrak{A}} X[6, \mathrm{p} .133]$. If $D$ is any subalgebra of $C(X)$ whose closure in $C(X)$ is equal to the closure of $\mathfrak{A} \mid X$ in $C(X)$, then we have $\partial_{\mathscr{D}} X=\partial_{\mathfrak{A}} X$. In particular,

$$
\partial_{\mathfrak{A}_{X}} X=\partial_{\mathfrak{A}} X .
$$


A necessary and sufficient condition for a point $\delta$ in $X$ to belong to $\partial_{\mathfrak{A}} X$ is that, for every neighborhood $V$ of $\delta$, there exist an element $f \in \mathfrak{A}$ with

$$
\sup _{\sigma \in X-V}|f(\sigma)|<\sup _{\sigma \in V}|f(\sigma)|=|f|_{X} .
$$

If such an $f$ exists with $|f(\delta)|=|f|_{x}$, then $\delta$ is called a strong boundary point of $X$ relative to $\mathfrak{A}$. In the case of a closed subalgebra of $C(X)$, strong boundary points are dense in the Silov boundary [6, p. 141]. Thus strong boundary points of $X$ relative to $\mathfrak{A}_{X}$ are dense in $\partial_{\mathfrak{U}} X$. As an immediate consequence of definitions, we have

$$
\partial_{\mathfrak{U}}\left(\text { hull }_{\mathfrak{U}} X\right)=\partial_{\mathfrak{U}} X \subseteq X .
$$

The Šilov boundary provides a kind of global maximum modulus principle for functions in $\mathfrak{A}_{X}$. Its existence depends only on the condition that the algebaa $\mathfrak{A}$ separate the points of $X$. On the other hand, since $\mathfrak{A}$ is assumed to be natural, a local maximum modulus principle within compact $\mathfrak{A}$-convex subsets of $\Sigma$ is valid. This, by Lemma 1.1, follows immediately from the local maximum modulus principle established by $H$. Rossi $[8,6.1$, p. 9]. We note in passing that the proof of Rossi's theorem depends in a nontrivial way on the theory of analytic functions of several complex variables. Now, for a statement of the local maximum-principle, let $\Omega$ denote a compact $\mathfrak{A}$ convex set in $\Sigma$ and let $\operatorname{bdry}_{2} U$ denote the topological boundary of $U$ (a subset of $\Omega$ ) with respect to the space $\Omega$.

1.3. Local maximum modulus principle. Let $U$ be a subset of $\Omega-\partial_{\mathfrak{A}} \Omega$ which is open in $\Omega$. Then, for every $f \in \mathfrak{A}$,

$$
\sup _{\omega \in V}|f(\omega)|=\sup _{\omega \in \text { bdry } \Omega}|f(\omega)| \text {. }
$$

Thus

$$
\partial_{\mathfrak{A}} \bar{U} \cong \operatorname{bdry}_{\Omega} U
$$

2. -holomorphic functions. Consider a complex-valued function $h$ defined on a subset $E$ of $\Sigma$. We say that $h$ is 2 -holomorphic at a point $\sigma \in E$ provided there exists a neighborhood $V$ of $\sigma$ such that $h$ is a uniform limit on $V \cap E$ of functions from $\mathfrak{A}$. Evidently if $h$ is $\mathfrak{A}$-holomorphic at $\sigma$ then it is $\mathfrak{A}$-holomorphic at every point of $E$ in the prescribed neighborhood $V$. Therefore the set of points at which $h$ is $\mathfrak{U}$-holomorphic is open relative to $E$. If $h$ is $\mathfrak{A}$-holomorphic at each point of $E$, then we say that it is 2 -holomorphic on (or $i n$ ) the set $E$. 
The class of all functions that are $\mathfrak{A}$-holomorphic on a given set

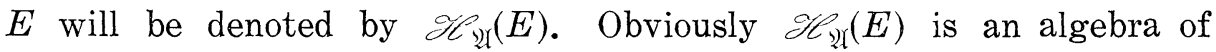
continuous functions on $E$. Note that $\mathscr{H}_{\mathscr{H}}(E)$ contains all functions on $E$ which belong locally to $\mathfrak{U}$ on $E$. We have $\mathfrak{U}_{E} \subseteq \mathscr{C}_{\mathcal{V}}(E)$, and the inclusion will in general be proper. In fact, even when $E$ is a compact $\mathfrak{A}$-convex set, there may exist functions which belong locally to $\mathfrak{A}$ on $E$ but which do not belong to $\mathfrak{A}_{E}$ [4, p. 822]. On the other hand, when $E$ is a compact $\mathfrak{A}$-convex set, the closure in $C(E)$ of the functions that belong locally to $\mathfrak{A}$ on $E$ is a natural algebra of functions on $E$ [10]. Whether or not the closure of $\mathscr{H}_{22}(E)$ in $C(E)$ is also natural under these conditions remains an open question.

We now establish some basic properties of $\mathfrak{A}$-holomorphic functions.

Lemma 2.1. If $h$ is $\mathfrak{2}$-holomorphic on a set $E$ and if $h(\xi) \neq 0$ for each $\xi \in E$, then $h^{-1}$ is also $\mathfrak{X}$-holomorphic on $E$.

Proof. Let $\sigma$ be an arbitrary point of $E$. Consider the disc

$$
D_{\rho}=\{\zeta:|\zeta-h(\sigma)|<\rho\},
$$

where $3 \rho=|h(\sigma)|$, and let $V$ be a neighborhood of $\sigma$ such that $h(\xi) \in D_{\rho}$ for $\xi \in V \cap E$ and $h$ is a uniform limit on $V \cap E$ of functions from $\mathfrak{A}$. Since $0 \notin D_{\rho}$, there exists, for arbitrary $\varepsilon>0$, a polynomial $P(\zeta)$ such that

$$
\left|P(\zeta)-\zeta^{-1}\right|<\frac{\varepsilon}{2} \quad \text { for } \zeta \in D_{\rho} .
$$

Choose $\delta$ with $0<\delta<\rho$ such that $\left|\zeta_{1}-\zeta_{2}\right|<\delta$ and $\zeta_{1}, \zeta_{2} \in D_{2 \rho}$ imply

$$
\left|P\left(\zeta_{1}\right)-P\left(\zeta_{2}\right)\right|<\frac{\varepsilon}{2} .
$$

Now choose $\alpha \in \mathfrak{A}$ such that

$$
|a(\xi)-h(\xi)|<\delta \quad \text { for } \xi \in V \cap E .
$$

Since $h(\xi) \in D_{\rho}$, it follows that $a(\xi) \in D_{2 \rho}$ and hence that

$$
\left|P(a)(\xi)-h^{-1}(\xi)\right|<\varepsilon \quad \text { for } \xi \in V \cap E .
$$

Thus $h^{-1}$ is $\mathfrak{A}$-holomorphic at $\sigma$ and the lemma is proved.

Next we show that a local maximum modulus principle is valid for $\mathfrak{A}$-holomorphic functions. (See also [2, proof Theorem 3.2].) 
THEOREM 2.2. Let $\Omega$ be a compact $\mathfrak{A}$-convex set in $\Sigma$ and let $U$ be a subset of $\Omega-\partial_{\mathfrak{2} \Omega}$ which is open in $\Omega$. If $h$ is a function which is continuous on $\bar{U}$ and $\mathfrak{A}$-holomorphic in $U$, then

$$
\sup _{\omega \in V}|h(\omega)|=\sup _{\omega \in \operatorname{bdry} \Omega U}|h(\omega)| \text {. }
$$

Proof. Consider the closed subalgebra $D$ of $C(\bar{U})$ generated by I) $\mid \bar{U}$ plus the function $h$. The theorem will follow if we show that $U \cap \partial_{\mathfrak{D}} \bar{U}=\varnothing$. Let $\omega_{0}$ be an arbitrary point of $U$ and choose a neighborhood $V$ of $\omega_{0}$ in $\Omega$ such that $\bar{V} \subset U$ and $h \mid \bar{V} \in \mathfrak{A}_{\bar{\nabla}}$. Then $\mathfrak{D} \mid \bar{V}=$ $\mathfrak{U}_{\bar{V}}$. Suppose $\omega_{0} \in \partial_{\mathscr{D}} \bar{U}$. Then there exists $f \in \mathfrak{D}$ and $\delta \in V$ such that

$$
|f(\delta)|>\sup _{\omega \in \bar{U}-V}|f(\omega)| \geqq \sup _{\omega \in \text { bdry }_{2} V}|f(\omega)| \text {. }
$$

But, since $f \mid \bar{V} \in \mathfrak{A}_{\bar{V}}$, this contradicts the local maximum modulus principle for $\mathfrak{A}$. Thus $U \cap \partial_{\mathfrak{D}} \bar{U}=\varnothing$ and the theorem is proved.

The result in Theorem 2.2 can be improved somewhat as follows. Suppose that $h$ is only defined and $\mathfrak{U}$-holomorphic in $U$. For arbitrary $\rho<|h|_{\sigma}$, set

$$
K_{\rho}=\overline{\{\omega: \omega \in U,|h(\omega)| \geqq \rho\}} .
$$

Then $K_{\rho}$ is nonvacuous, compact, contained in $\bar{U}$, and $\rho_{1}<\rho_{2}$ implies $K_{\rho_{2}} \subset K_{\rho_{1}}$. Therefore

$$
K=\bigcap_{\rho<|h|_{U}} K_{\rho} \neq \varnothing
$$

Observe that

$$
K \cap U=\left\{\omega: \omega \in U,|h(\omega)|=|h|_{U}\right\} .
$$

We prove that $K \cap \operatorname{bdry}_{\Omega} U \neq \varnothing$. If this were not true then there would exist an open set $W$ in $\Omega$ such that

$$
K \subset W \subset \bar{W} \subset U \text {. }
$$

Then $K \cap \operatorname{bdry}_{\Omega} W=\varnothing$. But, since $h \mid \bar{W} \in C(\bar{W})$, this contradicts Theorem 2.2. Therefore there exists $\delta \in K \cap \mathrm{bdry}_{\Omega} U$. If $V$ is any neighborhood of $\delta$, then $K_{\rho} \cap V \neq \varnothing$ for every $\rho<|h|_{U}$. Thus we obtain the following corollary.

CoRollary 2.3. Assume that $h$ is only defined and $\mathfrak{A}$-holomorphic within $U$. Then there exists $\delta \in \operatorname{bdry}_{\Omega} U$ such that, for every neighborhood $V$ of $\delta$,

$$
\sup _{\omega \in V}|h(\omega)|=\sup _{\omega \in V \cap U}|h(\omega)| .
$$


Applying the theorem to the case $U=\Omega-\partial_{\mathfrak{U}} \Omega$, we obtain the next corollary.

Corollary 2.4. Let $\Omega$ be any compact $\mathfrak{A}$-convex set in $\Sigma$. Then the Silov boundary of $\Omega$ relative to $\mathscr{K}_{\mathfrak{H}}(\Omega)$ is equal to $\partial_{\mathfrak{U}} \Omega$.

3. 2 -analytic varieties. Let $\Lambda$ and $\Omega$ be subsets of $\Sigma$. The set $\Lambda$ will be called an 2 -analytic variety within $\Omega$ if $A \subseteq \Omega$ and for each $\lambda \in \Lambda$ there exists a neighborhood $V$ of $\lambda$ such that $V \cap A$ is an intersection of zero sets of functions (possibly infinite in number) which are $\mathfrak{U}$-holomorphic in $V \cap \Omega$. In other words, if $\omega \in V \cap \Omega-\Lambda$, then there exists a function $h$ which is $\mathfrak{U}$-holomorphic on $V \cap \Omega$ and vanishes on $V \cap A$ but not at the point $\omega$. It is obvious that, if $\Gamma \subseteq \Omega$, then $\Lambda \cap I$ is an $\mathfrak{A}$-analytic variety within $\Gamma$. If $A$ is relatively closed in $\Omega$, then it will be called an $\mathfrak{A}$-analytic subvariety of $\Omega$. If $V \cap A$ is the zero set of a single function $\mathfrak{A}$-holomorphic in $V \cap \Omega$, then $\Lambda$ is called an $\mathfrak{A}$-analytic hypersurface. Trivial examples of $\mathfrak{A}$-analytic subvarieties (actually hypersurface) of a given set $\Omega$ are the empty set and the set $\Omega$ itself.

We establish next an important lemma for the proof of the main theorem concerning varieties. It's proof can also be adapted to give a related lemma obtained by Glicksberg [2, Lemma 2.1] for quite a different purpose. It is convenient to make another definition before stating the lemma. Let $X$ and $\Omega$ be subsets of $\Sigma$ with $X \subseteq \Omega$ and let $\delta \in \Omega$. Then the set $X$ is said to be locally determining in $\Omega$ at $\delta$ if for every neighborhood $U$ of $\delta$ there exists a neighborhood $V$ of $\delta$ such that $V \cong U$ and any function which is $\mathfrak{U}$-holomorphic on $U \cap \Omega$ and vanishes on $U \cap X$ must also vanish on $V \cap \Omega$.

Lemma 3.1. Let $\Omega=\operatorname{hull}_{\mathfrak{U}} X$ where $X$ is a compact set in $\Sigma$. If $X \neq \Omega$, then there exists a point $\delta \in \operatorname{bdry}_{\Omega} X$ at which $X$ is locally determining in $\Omega$.

Proof. Set $G=\Omega-X$. Then $G$ is open in $\Omega$. If $D$ denotes the closure of the algebra $\mathfrak{U} \mid G$ in $C(\bar{G})$, then by the local maximum modulus principle for $\mathfrak{A}$, we have

$$
\partial_{\mathscr{D}} \bar{G}=\partial_{\mathscr{Y}} \bar{G} \cong \operatorname{bdry}_{\Omega} G=\operatorname{bdry}_{\Omega} X .
$$

Let $\delta$ be a strong boundary point of $\bar{G}$ relative to $\mathfrak{D}$. Then $\delta \in \operatorname{bdry}_{\Omega} X$ and we will show that $X$ is locally determining in $\Omega$ at $\delta$. Let $U$ be an arbitrary open neighborhood of the point $\delta$. Then there exists $b \in \mathfrak{D}$ such that 


$$
\sup _{\omega \in \bar{G}-U}|b(\omega)|<|b(\delta)|=|b|_{\bar{\alpha}} .
$$

Since $\mathfrak{A} \mid \bar{G}$ is dense in $\mathfrak{D}$, it is straightforward to obtain $u \in \mathfrak{A}$ such that

$$
\sup _{\omega \in \bar{G}-U}|u(\omega)|<\frac{1}{3}, \quad|u(\delta)|>1
$$

Define

$$
V=\{\omega: \omega \in U,|u(\omega)|>1\} .
$$

Then $V$ is an open neighborhood of $\delta$ and $V \subseteq U$. Also let

$$
W=\left\{\omega: \omega \in U,|u(\omega)|<\frac{2}{3}\right\} .
$$

Then $V \cong W \cong U$ and $\bar{W} \cap \bar{G} \subset U \cap \bar{G}$.

Now suppose that $h$ were $\mathfrak{Y}$-holomorphic on $U \cap \Omega$ and that $h(U \cap X)=(0)$ while $h(\sigma) \neq 0$ for some $\sigma \in V \cap \Omega$. Since $h$ is continuous on $U \cap \Omega$ and zero on $U \cap X$, it follows that $h$ is bounded on $\bar{W} \cap \bar{G}$ and

$$
|h|_{\bar{W} \cap \bar{\theta}}=|h|_{\bar{w} \cap \Omega} .
$$

Hence there exists an integer $m$ such that

$$
\left(\frac{2}{3}\right)^{m}|h|_{\bar{w} \cap \Omega}<|h(\sigma)| \text {. }
$$

Define

$$
g(\omega)=u(\omega)^{m} h(\omega), \quad \omega \in U \cap \Omega .
$$

Then $g$ is also $\mathfrak{A}$-holomorphic on $U \cap \Omega$ and $g(U \cap X)=(0)$. Since $|u(\sigma)|>1$, we have

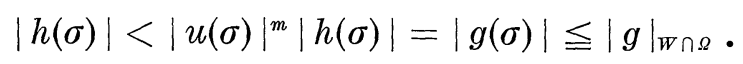

By the local maximum modulus principle for $\mathfrak{A}$-holomorphic functions (Theorem 2.2) and the fact that $g$ is $\mathfrak{A}$-holomorphic on $\bar{W} \cap \Omega$, there must exist a point $\tau \in \operatorname{bdry}_{\Omega}(W \cap \Omega)$ such that

$$
|h(\sigma)|<|g(\tau)| \text {. }
$$

Note that $\tau$ must belong to $\bar{G}$ since $g(U \cap X)=(0)$. From the definition of $W$ and the fact that $\bar{W} \cap \bar{G} \subset U \cap \bar{G}$, it follows that $|u(\tau)|=$ $2 / 3$. Therefore

$$
|g(\tau)|=\left(\frac{2}{3}\right)^{m}|h(\tau)| \leqq\left(\frac{2}{3}\right)^{m}|h|_{\bar{w} \cap \Omega}<|h(\sigma)| .
$$


This is a contradiction and completes the proof of the lemma.

We are now ready to prove the main theorem concerning convexity properties of $\mathfrak{A}$-analytic varieties.

TheoRem 3.2. Let $\Omega$ be any compact $\mathfrak{A}$-convex set in $\Sigma$. Then every $\mathfrak{A}$-analytic subvariety of $\Omega$ is $\mathfrak{A}$-convex.

Proof. Let $\Lambda$ be an $\mathfrak{U}$-analytic subvariety of $\Omega$ and set

$$
\Delta=\operatorname{hull}_{\mathfrak{A}} \Lambda \text {. }
$$

Since $\Omega$ is 2 -convex and $A \subseteq \Omega$, we also have $\Delta \subseteq \Omega$. The problem is to show that $A=\Delta$. From the compactness of $\Omega$ and the fact that $A$ is closed in $\Omega$, it follows that $A$ is also compact. Hence, if $\Lambda \neq \Delta$, then Lemma 3.1 applies giving a point $\delta \in \operatorname{bdry}_{\Delta} \Lambda$ at which $A$ is locally determining in $\Delta$. Since $A$ is closed, $\delta \in A$ and there exists a neighborhood $U$ of $\delta$ such that $U \cap A$ is an intersection of zero sets of functions $\mathfrak{A}$-holomorphic in $U \cap \Omega$. Now choose a neighborhood $V$ of $\delta$ in accordance with the local determining property. Since a function which is $\mathfrak{A}$-holomorphic in $U \cap \Omega$ is automatically $\mathfrak{A}$ holomorphic in $U \cap \Delta$, it follows that any function which is $\mathfrak{A}$-holomorphic in $U \cap \Omega$ and zero on $U \cap A$ will also be zero on $V \cap \Delta$. Therefore $V \cap \Delta \subset \Lambda$. But this is impossible since $V$ is a neighborhood of a boundary point of $A$ within the space $\Delta$. Hence we must have $\Lambda=\Delta$, completing the proof of the theorem.

Since a closed polynomial polyhedron in $\boldsymbol{C}^{n}$ is polynomially convex we have the following corollary for $\boldsymbol{C}^{n}$.

COROLLARY 3.3. Every analytic subvariety of a closed polynomial. polyhedron in $C^{n}$ is polynomially convex.

We obtain next a generalization of another version of the result for $C^{n}\left[9,\left(\right.\right.$ A. 21) p. 285]. For an arbitrary finite set $\left\{a_{1}, \cdots, a_{m}\right\}$ of elements of $\mathfrak{A}$, the set

$$
\Theta=\left\{\sigma: \sigma \in \Sigma,\left|a_{i}(\sigma)\right|<1,(i=1, \cdots, m)\right\}
$$

will be called an open $\mathfrak{A}$-polyhedron in $\Sigma$. (If “ $<$ " is replaced by “ฏ”, then $\Theta$ is a closed $\mathfrak{A}$-polyhedron.)

THEOREM 3.4. Let $\Lambda$ be an $\mathfrak{A}$-analytic subvariety of an open $\mathfrak{A}$-polyhedron $\Theta$ in $\Sigma$. Then $X \subseteq \Lambda$ implies hull $\mathfrak{A} \subseteq \subseteq \Lambda$, for $X$ any compact set. 
Proof. Let $\Theta$ be defined by the elements $a_{1}, \cdots, a_{m} \in \mathfrak{A}$ and let $X$ be any compact subset of $\Lambda$. Then hull $_{\mathfrak{A}} X$ is also compact. If $\sigma \in \operatorname{hull}_{\mathfrak{A}} X$, then

$$
|a(\sigma)| \leqq|a|_{x}, \quad a \in \mathfrak{A} .
$$

In particular, since $X \subseteq A \leqq \Theta$,

$$
\left|a_{i}(\sigma)\right| \leqq\left|a_{i}\right|_{x}<1, \quad i=1, \cdots, m .
$$

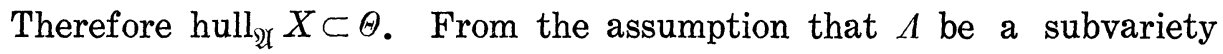
of $\Theta$ we have that $A$ is relatively closed in $\Theta$ and so $A \cap\left(\right.$ hull $\left._{\mathfrak{A}} X\right)$ is closed. In other words, $A \cap\left(\right.$ hull $\left._{\mathfrak{A}} X\right)$ is a subvariety of hull $\mathscr{\mathfrak { A }} X$. Hence, by Theorem $3.2, \Lambda \cap\left(\right.$ hull $\left._{\mathfrak{A}} X\right)$ is $\mathfrak{A}$-convex. Since $X \subseteq \Lambda \cap$ $\left(\right.$ hull $\left._{\mathfrak{A}} X\right)$, it follows that

$$
\operatorname{hull}_{\mathfrak{A}} X=\Lambda \cap\left(\operatorname{hull}_{\mathfrak{A}} X\right) \subseteq \Lambda,
$$

and the theorem is proved.

The next theorem generalizes a result of Oka in which an analytic polyhedron in $\boldsymbol{C}^{n}$ is represented as a polynomial convex set in higher dimension. [5, p. 21; 1, p. 115]. We must first extend the concept of analytic polyhedron to the general case. Let $G$ be an open set in $\Sigma$ and let $h_{1}, \cdots, h_{m}$ be $\mathfrak{X}$-holomorphic in $G$. Set

$$
\Delta=\left\{\sigma: \sigma \in G,\left|h_{i}(\sigma)\right| \leqq 1, i=1, \cdots, m\right\} .
$$

Then $\Delta$ will be called an $\mathfrak{A}$-analytic polyhedron in $G$. Now consider the product space $\Sigma \times C^{m}$ consisting of all pairs $(\sigma, \zeta)$ where $\sigma \in \Sigma$ and

$$
\zeta=\left(\zeta_{1}, \cdots, \zeta_{m}\right) \in C^{m}
$$

Let

$$
\Gamma=\left\{(\sigma, \zeta): \sigma \in \Delta, \zeta=\left(h_{1}(\sigma), \cdots, h_{m}(\sigma)\right)\right\} .
$$

Thus $\Gamma$ is the "graph" in $\Sigma \times C^{m}$ of the vector-valued function $\zeta(\cdot)$, where

$$
\zeta(\sigma)=\left(h_{1}(\sigma), \cdots, h_{m}(\sigma)\right), \quad \sigma \in \Delta .
$$

If $\Pi$ denotes the unit polydisc in $C^{m}$, then

$$
\Gamma \leqq \Delta \times \Pi \subset G \times C^{m}
$$

Note that $G \times C^{m}$ is an open set in $\Sigma \times C^{m}$. If $\Delta$ is a closed set in $\Sigma$, then it is not difficult to verify that $\Gamma$ is a closed set in $\Sigma \times C^{m}$. Next let $\mathscr{P}_{\mathfrak{A}}$ denote the algebra of all polynomials in $m$ complex 
variables $\zeta_{1}, \cdots, \zeta_{m}$ with coefficients in $\mathfrak{A}$. Each $P \in \mathscr{P}_{\mathfrak{H}}$ can be regarded as a function defined on $\Sigma \times \boldsymbol{C}^{m}$ of the form

$$
P(\sigma, \zeta)=\sum a_{k_{1}, \ldots, k_{m}}(\sigma) \zeta_{1}^{k_{1}} \cdots \zeta_{\zeta_{m}}^{k_{m}},
$$

where the number of terms in the sum is finite and each coefficient $a_{k_{1}, \ldots, k_{m}}$ belongs to $\mathfrak{A}$. It is a routine matter to show that $\mathscr{P}_{\mathfrak{U}}$ is a natural algebra of functions on $\Sigma \times \boldsymbol{C}^{m}$. Furthermore, if we define

$$
H_{i}(\sigma, \zeta)=h_{i}(\sigma)-\zeta_{i}
$$

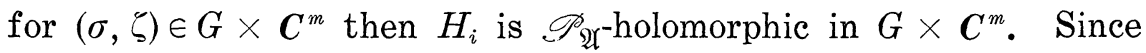

$$
\Gamma=\left\{(\sigma, \zeta):(\sigma, \zeta) \in G \times C^{m}, H_{i}(\sigma, \zeta)=0, i=1, \cdots, m\right\}
$$

we see that $\Gamma$ is a $\mathscr{P}_{\mathfrak{2}}$-analytic variety within $G \times \boldsymbol{C}^{m}$. We can now prove the generalization of Oka's theorem. The latter is obtained from the general theorem in the usual way by taking $\Sigma=\boldsymbol{C}^{n}$ and $\mathfrak{A}=\mathscr{P}$.

THEOREM 3.5. If the $\mathfrak{A}$-analytic polyhedron $\Delta$ is compact, then its associated graph $\Gamma$ is $\mathscr{P}_{\mathfrak{U}^{-}}$-convex in $\Sigma \times \boldsymbol{C}^{m}$.

Proof. Let $\Omega$ denote hull $\Delta$ in $\Sigma$. Then $\Omega$ is compact. Consider the compact set $\Omega \times \Pi$ in $\Sigma \times C^{m}$. We prove that $\Omega \times \Pi$ is $\mathscr{P}_{\mathfrak{y}}$-convex. Let

$$
(\delta, \xi) \in \operatorname{hull}_{\mathscr{P}, \mathfrak{Z}}(\Omega \times \Pi)
$$

and consider the function

$$
Z_{i}(\sigma, \zeta)=\zeta_{i}, \quad(\sigma, \zeta) \in \Sigma \times C^{m} .
$$

Then $Z_{i} \in \mathscr{P}_{\mathcal{W}}$ for each $i=1, \cdots, m$ so we have

$$
\left|\xi_{i}\right|=\left|Z_{i}(\delta, \xi)\right| \leqq\left|Z_{i}\right|_{\Omega \times \Pi}=\sup _{\xi \in \Pi}\left|\zeta_{i}\right|=1 .
$$

It follows that $\xi \in \Pi$. Next consider, for $a \in \mathfrak{A}$, the function

$$
A(\sigma, \zeta)=a(\sigma), \quad(\sigma, \zeta) \in \Sigma \times C^{m} .
$$

Then $A \in \mathscr{P}_{\mathfrak{U}}$ and hence

$$
|a(\delta)|=|A(\delta, \xi)| \leqq|A|_{\Omega \times \Pi}=\sup _{\sigma \in \Omega}|a(\sigma)| .
$$

Therefore $\delta \in \Omega$. In other words, $(\delta, \xi) \in \Omega \times \Pi$ so $\Omega \times \Pi$ is $\mathscr{P}_{\mathscr{H}^{-}}$ convex. We now have $\Gamma$ as a $\mathscr{P}_{2}$-analytic subvariety of $\Omega \times \Pi$. Therefore, by Theorem 3.2, $\Gamma$ is $\mathscr{P}_{2}$-convex and the theorem is proved. 
4. A local property of $\mathfrak{A}$-convex sets. Let $\Omega$ be a compact $\mathfrak{A}$-convex set in $\Sigma$ and let $U$ be a subset of $\Omega-\partial_{\mathfrak{A}} \Omega$ which is open in the space $\Omega$. Denote by $\mathscr{F}$ a family of functions each of which is continuous on $\bar{U}$ and $\mathfrak{A}$-holomorphic in $U$. For each $f \in \mathscr{F}$ set

$$
\Lambda_{f}=\{\omega: \omega \in \bar{U}, f(\omega)=0\} \text {. }
$$

In this situation we have the following lemma.

Lemma 4.1. Assume that $\mathscr{F}$ is a connected subset of $C(\bar{U})$ and that there exist $g, h \in \mathscr{F}$ for which

$$
\Lambda_{g} \cap U \neq \varnothing \quad \text { and } \quad \Lambda_{h} \cap U=\varnothing .
$$

Then there also exists $k \in \mathscr{F}$ for which

$$
\Lambda_{k} \cap \operatorname{bdry}_{\Omega} U \neq \varnothing \text {. }
$$

Proof. Consider the collection of all those functions which are continuous on $\bar{U}$ and $\mathfrak{A}$-holomorphic in $U$. This is a subalgebra of $C(\bar{U})$ whose closure in $C(\bar{U})$ we denote by $\mathfrak{D}$. Then $\mathscr{F} \cong \mathfrak{D}$ and, by Theorem 2.2,

$$
\partial_{\mathscr{D}} \bar{U} \cong \operatorname{bdry}_{\Omega} U
$$

Identify $\bar{U}$ in the usual way with its natural embedding in the carrier space $\Phi_{\mathscr{D}}$ of the Banach algebra $\mathfrak{D}[6, \mathrm{p} .120]$. Then we have

$$
\partial_{\mathscr{D}} \Phi_{\mathscr{D}} \subseteq \partial_{\mathscr{D}} \bar{U} \subseteq \operatorname{bdry}_{\Omega} U
$$

Observe that, by Lemma 2.1, a function which is continuous on $\bar{U}$ and $\mathfrak{A}$-holomorphic in $U$ will be singular in $\mathfrak{D}$ if and only if it has a zero in $\bar{U}$.

Now let $\mathscr{F}_{s}$ denote those elements of $\mathscr{F}$ that are singular in $\mathscr{D}$ and $\mathscr{F}_{r}$ those that are regular in $\mathfrak{D}$. Then $g \in \mathscr{F}_{s}$ so that $\mathscr{F}_{s}$ is not empty. In case $\mathscr{F}_{s}=\mathscr{F}$, then in particular the element $h$ is singular and accordingly must have a zero in $\bar{U}$. Since $\Lambda_{h} \cap U=\varnothing$, it follows that $\Lambda_{h} \cap$ bdry $_{\Omega} U \neq \varnothing$, so we can take $k=h$ in this case. In case $\mathscr{F}_{s} \neq \mathscr{F}$, then both $\mathscr{F}_{s}$ and $\mathscr{F}_{r}$ are nonempty. Since $\mathscr{F}$ is connected in $C(\bar{U})$, it follows that either $\overline{\mathscr{F}}_{s} \cap \mathscr{F}_{r} \neq \varnothing$ or $\mathscr{F}_{s} \cap \overline{\mathscr{F}}_{r} \neq \varnothing$. From the fact that the singular elements constitute a closed set in a Banach algebra, we conclude that the first possibility cannot occur. Hence $\mathscr{F}_{s} \cap \overline{\mathscr{F}}_{r} \neq \varnothing$. In other words, $\mathscr{F}_{s}$ contains an element $k$ which is a limit of regular elements. But then $k$ is a topological divisor of zero in $\mathscr{D}[6$, p. $22 ; 7$, p. 1066], and therefore its image in the Gelfand representation of $\mathfrak{D}$ must have a zero on the Šilov boundary, 
$\partial_{\mathscr{D}} \Phi_{\mathscr{D}}\left[6\right.$, p. $137 ; 7$, p. 1076]. Since $\partial_{\mathscr{D}} \Phi_{\mathscr{D}} \subseteq \mathrm{bdry}_{\Omega} U$, it follows that $A_{k} \cap \operatorname{bdry}_{\Omega} U \neq \varnothing$ and the proof is complete.

We obtain next the local property of $\mathscr{A}$-convex hulls in $\Sigma$ which generalizes the Oka result for polynomial convex hulls in $C^{n}$ mentioned in the Introduction. This involves the concept of a continuous family of $\mathfrak{A}$-analytic hypersurfaces which we now describe. Let $G$ be an open set in $\Sigma$. For each $t \in[0,1]$ let $h_{t}$ be a function which is $\mathfrak{A}$ holomorphic in $G$ and consider the hypersurface

$$
\Lambda_{t}=\left\{\sigma: \sigma \in G, h_{t}(\sigma)=0\right\} .
$$

We say that $\left\{\Lambda_{t}\right\}$ is a continuous family of hypersurfaces within $G$ if the mapping $(t, \sigma) \rightarrow h_{t}(\sigma)$ of $[0,1] \times G$ into the complex plane is continuous. The family is said to intersect a set $X$ if $A_{t} \cap X \neq \varnothing$ for some $t$. It is said to intersect $X$ nontrivially if $A_{t} \cap X$ is closed relative to $X$ for each $t$ and the set $\left\{t: \Lambda_{t} \cap X \neq \varnothing\right\}$ is a proper, closed, nonempty subset of $[0,1]$. In the case of $C^{n}$, Oka [5, p. 14] calls an analytic hypersurface a "characteristic surface" and Stoltzenberg $[9$, p. 264] calls a continuous family of analytic hypersurfaces a "curve" of analytic hypersurfaces.

THEOREM 4.2. Let $X$ be a compact subset of $\Sigma$. Then every continuous family of $\mathfrak{U}$-analytic hypersurfaces which intersects hull $_{\mathfrak{A}} X$ nontrivially must intersect $X$.

Proof. Set $\Omega=\operatorname{hull}_{\mathfrak{2}} X$ and let $\left\{\Lambda_{t}: t \in[0,1]\right\}$ be a continuous family of hypersurfaces within the open set $G$. Assume that $\left\{\Lambda_{t}\right\}$ intersects $\Omega$ nontrivially but that it does not intersect $X$. Consider the set

$$
T=\left\{t: \Lambda_{t} \cap \Omega \neq \varnothing\right\} .
$$

By hypothesis, $T$ is a proper, closed, nonempty subset of $[0,1]$ and therefore its complement in $[0,1]$ must contain an open interval with at least one end-point in the set $T$. Hence, by a simple change of parameter $t$, we can reduce to the case in which $A_{0} \cap \Omega \neq \varnothing$ and $\Lambda_{t} \cap \Omega=\varnothing$ for $0<t<1$. Since $\Lambda_{0} \cap \Omega$ is compact and contained in $G-X$, there exists a relatively open subset $U$ of $\Omega$ such that

$$
\bar{U} \subset G-X \quad \text { and } \quad \Lambda_{t} \cap \Omega \subset U
$$

for all $t \in[0,1]$. Let $\left\{h_{t}: t \in[0,1]\right\}$ be the family of $\mathfrak{A}$-holomorphic functions which determines the family $\left\{\Lambda_{t}\right\}$. Then each $h_{t}$ is $\mathfrak{X}$ holomorphic in $G$ and hence on the set $\bar{U}$. Also, by the continuity condition, it is obvious that the functions $h_{t}$ restricted to $\bar{U}$ con- 
stitute a connected subset of $C(\bar{U})$. Therefore, by Lemma 4.1,

$$
\Lambda_{t} \cap \operatorname{bdry}_{\Omega} U \neq \varnothing
$$

for some $t$. Since $\Lambda_{t} \cap \Omega \subset U$, this is impossible so the theorem follows.

We are indebted to John Garnett for pointing out that Theorem 4.2 is actually false if in the definition of nontrivial intersection we drop the requirement that the set $T$ be closed. Furthermore, the result for $C^{n}$ claimed by Oka [5, p. 14] is also false. However, Oka's proof does yield a result for $C^{n}$ which may easily be deduced from Theorem 4.2.

Although the result for $C^{n}$ follows from Theorem 4.2, it should be noted that the proof of Theorem 4.2 involves the local maximum modulus principle whose proof in turn involves results from the theory of analytic functions of several complex variables as deep as those involved in the proof of the Oka theorem. In fact, one of the nicest proofs of the local maximum principle can be obtained from the Oka theorem [9, p. 265]. Similarly one can easily deduce the general local maximum principle from the property of 2 -convex hulls proved in Theorem 4.2. In other words these two properties of $\mathfrak{U}$-convex hulls are equivalent.

Lemma 4.1 will now be used to obtain some further properties of $\mathfrak{A}$-holomorphic functions. These properties, in the case of functions in $\mathfrak{A}$, can be obtained from known results for Banach algebras of functions [6, Section 3.3]. The first is a partial extension of Rouché's theorem. For functions in $\mathfrak{A}$, Theorems 4.3 and 4.4 follow from theorems due to John Holladay [3; 6, (3.3.22), (3.3.23)]. As before, $\Omega$ will denote a compact $\mathfrak{A}$-convex set in $\Sigma$ and $U$ will be a subset of $\Omega-\partial_{\mathfrak{A}} \Omega$ which is open in $\Omega$.

THEOREM 4.3. Let $f$ and $g$ be continuous on $\bar{U}$ and 2 -holomorphic in $U$. If

$$
|f(\omega)-g(\omega)|<|f(\omega)| \quad \text { for } \omega \in \operatorname{bdry}_{\Omega} U,
$$

then $f$ will have a zero in $U$ if and only if $g$ does.

Proof. Consider the family of functions

$$
h_{t}=(1-t) f+t g, \quad t \in[0,1] .
$$

Then each $h_{t}$ is continuous on $\bar{U}$ and $\mathfrak{A}$-holomorphic in $U$. Obviously $\left\{h_{t}\right\}$ is a connected subset of $C(\bar{U})$. Furthermore, for arbitrary $\omega \in$ $\operatorname{bdry}_{\Omega} U$ and $t \in[0,1]$, 


$$
\begin{aligned}
0<|f(\omega)|-|f(\omega)-g(\omega)| & \leqq|f(\omega)|-t|f(\omega)-g(\omega)| \\
& \leqq|f(\omega)-t[f(\omega)-g(\omega)]| \\
& =\left|h_{t}(\omega)\right| .
\end{aligned}
$$

Hence $h_{t}$ does not have a zero on $\operatorname{bdry}_{\Omega} U$. Therefore, by Lemma 4.1, either every $h_{t}$ has a zero in $U$ or no $h_{t}$ has a zero in $U$. Since $h_{0}=f$ and $h_{1}=g$, the theorem follows.

THEOREM 4.4. Let $f$ be continuous on $\bar{U}$ and $\mathfrak{A}$-holomorphic in $U$. Let $\omega_{0}$ be a point of $U$ and define

$$
\delta=\min _{\omega \in \text { bdry } \Omega U}\left|f(\omega)-f\left(\omega_{0}\right)\right| .
$$

If $\delta>0$, then $f(U)$ contains the disc

$$
\left\{\zeta: \zeta \in \boldsymbol{C},\left|\zeta-f\left(\omega_{0}\right)\right|<\delta\right\} .
$$

Proof. Consider the family $\mathscr{F}$ of functions of the form $f_{\zeta}=$ $f-\zeta$, where $\zeta$ is an arbitrary point of the given disc. Then each $f_{\zeta}$ is continuous on $\bar{U}$ and $\mathfrak{A}$-holomorphic in $U$. Obviously $\mathscr{F}$ is a connected subset of $C(\bar{U})$. Furthermore, if $\left|\zeta-f\left(\omega_{0}\right)\right|<\delta$ and $\omega \epsilon$ bdry $U$, then

$$
\begin{aligned}
\left|f_{\zeta}(\omega)\right| & \geqq\left|f(\omega)-f\left(\omega_{0}\right)\right|-\left|f\left(\omega_{0}\right)-\zeta\right| \\
& \geqq \delta-\left|f\left(\omega_{0}\right)-\zeta\right|>0 .
\end{aligned}
$$

Therefore none of the functions in $\mathscr{F}$ has a zero on $\operatorname{bdry}_{\Omega} U$. Since $f_{\zeta}\left(\omega_{0}\right)=0$ for $\zeta=f\left(\omega_{0}\right)$, it follows from Lemma 4.1 that every function $f_{\zeta}$ has a zero in $U$. In other words, $\zeta \in f(U)$ for every $\zeta$ in the disc.

THEOREM 4.5. If $f$ is continuous on $\bar{U}$ and $\mathfrak{A}$-holomorphic in $U$, then

$$
\operatorname{bdry}_{C} f(U) \subseteq f\left(\operatorname{bdry}_{\Omega} U\right)
$$

Proof. Let $\zeta_{0}$ be a boundary point of $f(U)$ and set

$$
3 \delta=\min _{\omega \in \operatorname{bdry} \Omega U}\left|f(\omega)-\zeta_{0}\right| \text {. }
$$

Suppose $\zeta_{0} \notin f\left(\operatorname{bdry}_{\Omega} U\right)$. Then $\delta>0$. Since $\zeta_{0}$ is on the boundary of $f(U)$, there exists $\omega_{0} \in U$ such that $\left|f\left(\omega_{0}\right)-\zeta_{0}\right|<\delta$. We then have

$$
\min _{\omega \in \operatorname{bdry} \Omega}\left|f(\omega)-f\left(\omega_{0}\right)\right| \geqq 2 \delta \text {. }
$$

Hence, by the preceding theorem 


$$
\left\{\zeta:\left|\zeta-f\left(\omega_{0}\right)\right|<2 \delta\right\} \subset f(U)
$$

Since

$$
\begin{aligned}
\left|\zeta-f\left(\omega_{0}\right)\right| & \leqq\left|\zeta-\zeta_{0}\right|+\left|\zeta_{0}-f\left(\omega_{0}\right)\right| \\
& <\left|\zeta-\zeta_{0}\right|+\delta,
\end{aligned}
$$

it follows that

$$
\left\{\zeta:\left|\zeta-\zeta_{0}\right|<\delta\right\} \subset f(U)
$$

But this is impossible because $\zeta_{0} \in \operatorname{bdry}_{C} f(U)$. Therefore we must have $\zeta_{0} \in f\left(\operatorname{bdry}_{\Omega} U\right)$ and the theorem follows.

\section{BIBLIOGRAPHY}

1. L. Bers, Introduction to several complex variables, Mimeographed notes, New York University (1964).

2. I. Glicksberg, Maximal algebras and a theorem of Rado, Pacific J. Math. 14 (1964), 919-941.

3. J. C. Holladay, Boundary conditions for algebras of continuous functions, Ph. D. Dissertation, Yale University (1953).

4. Eva Kallin, A nonlocal function algebra, Proc. Nat. Acad. Sc1. 49 (1963), 821-824.

5. K. Oka, Sur les fonctions analytiques de plusieurs variables, Iwanami Shoten, Tokyo, 1961.

6. C. E. Rickart, General theory of Banach algebras, Van Nostrand, Princeton, (1960). 7. - The singular elements of a Banach algebra, Duke Math. J. 14 (1947), 1063-1077.

8. H. Rossi, The local maximum modulus principle, Ann. of Math. 72 (1960), 1-11.

9. G. Stolzenberg, Polynomially and rationally convex sets, Acta Mathematica 109 (1963), 259-289.

10. The maximal ideal space of the functions locally in a function algebra, Proc. Amer. Math. Soc. 14 (1963), 342-345.

Received February 15, 1965. This research was partially supported by the Air Force Office of Scientific Research under grant AFOSR-407-63.

YALE UNIVERSITY 



\section{PACIFIC JOURNAL OF MATHEMATICS}

\section{EDITORS}

H. SAMELSON

Stanford University

Stanford, California

R. M. BLUMENTHAL

University of Washington

Seattle, Washington 98105

\section{*J. DugundJI}

University of Southern California Los Angeles, California 90007

RICHARD ARENS

University of California

Los Angeles, California 90024

\section{ASSOCIATE EDITORS}

E. F. BECKENBACH
F. WOLF

K. YosIDA

\section{SUPPORTING INSTITUTIONS}

\author{
UNIVERSITY OF BRITISH COLUMBIA \\ CALIFORNIA INSTITUTE OF TECHNOLOGY \\ UNIVERSITY OF CALIFORNIA \\ MONTANA STATE UNIVERSITY \\ UNIVERSITY OF NEVADA \\ NEW MEXICO STATE UNIVERSITY \\ OREGON STATE UNIVERSITY \\ UNIVERSITY OF OREGON \\ OSAKA UNIVERSITY \\ UNIVERSITY OF SOUTHERN CALIFORNIA
}

\author{
STANFORD UNIVERSITY \\ UNIVERSITY OF TOKYO \\ UNIVERSITY OF UTAH \\ WASHINGTON STATE UNIVERSITY \\ UNIVERSITY OF WASHINGTON \\ AMERICAN MATHEMATICAL SOCIETY \\ CHEVRON RESEARCH CORPORATION \\ TRW SYSTEMS \\ NAVAL ORDNANCE TEST STATION
}

Mathematical papers intended for publication in the Pacific Journal of Mathematics should be typewritten (double spaced). The first paragraph or two must be capable of being used separately as a synopsis of the entire paper. It should not contain references to the bibliography. Manuscripts may be sent to any one of the four editors. All other communications to the editors should be addressed to the managing editor, Richard Arens at the University of California, Los Angeles, California 90024 .

50 reprints per author of each article are furnished free of charge; additional copies may be obtained at cost in multiples of 50 .

The Pacific Journal of Mathematics is published monthly. Effective with Volume 16 the price per volume (3 numbers) is $\$ 8.00$; single issues, $\$ 3.00$. Special price for current issues to individual faculty members of supporting institutions and to individual members of the American Mathematical Society: $\$ 4.00$ per volume; single issues $\$ 1.50$. Back numbers are available.

Subscriptions, orders for back numbers, and changes of address should be sent to Pacific Journal of Mathematics, 103 Highland Boulevard, Berkeley 8, California.

Printed at Kokusai Bunken Insatsusha (International Academic Printing Co., Ltd.), No. 6, 2-chome, Fujimi-cho, Chiyoda-ku, Tokyo, Japan.

PUBLISHED BY PACIFIC JOURNAL OF MATHEMATICS, A NON-PROFIT CORPORATION

The Supporting Institutions listed above contribute to the cost of publication of this Journal, but they are not owners or publishers and have no responsibility for its content or policies.

* Paul A. White, Acting Editor until J. Dugundji returns. 


\section{Pacific Journal of Mathematics}

\section{Vol. 18, No. 2 \\ April, 1966}

Alexander V. Arhangelskii, On closed mappings, bicompact spaces, and a problem of P. Aleksandrov .............................. 201

A. K. Austin, A note on loops . . . . . . . . . . . . . . . . . . . . . . . . . . . . 209

Lawrence Peter Belluce and William A. Kirk, Fixed-point theorems for families of contraction mappings...................... 213

Luther Elic Claborn, Every abelian group is a class group ............ 219

Luther Elic Claborn, A note on the class group .................. 223

Robert Stephen De Zur, Point-determining homomorphisms on multiplicative semi-groups of continuous functions............. 227

Raymond William Freese, A convexity property ................ 237

Frederick Paul Greenleaf, Characterization of group algebras in terms of their translation operators ......................... 243

Andrzej Hulanicki, On the spectral radius of hermitian elements in group algebras....................................... 277

Michael Bahir Maschler and Bezalel Peleg, A characterization, existence proof and dimension bounds for the kernel of a game ............ 289

Yiannis (John) Nicolas Moschovakis, Many-one degrees of the predicates

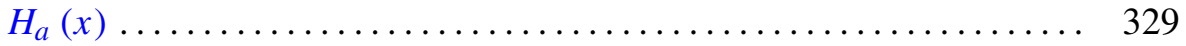

G. O. Okikiolu, $n$th order integral operators associated with Hilbert transforms.

C. E. Rickart, Analytic phenomena in general function algebras ... 361

K. N. Srivastava, On an entire function of an entire function defined by Dirichlet series

Paul Elvis Waltman, Oscillation criteria for third order nonlinear differential equations .......................... 